\title{
Transmission-type Window of HFCVD Diamond Film for Microfocus X-ray Tube
}

\author{
Lei Zheng, Huarong Liu and Junting Wang \\ No 38 Research Institute of CETC, Hefei 230088, China
}

\begin{abstract}
Operating the microfocus X-ray source at high power is required to achieve high temporal resolution. However, the thermal loading of the anode focal spot is a limiting factor in determining the maximum power of an x-ray tube. In this paper, a transmission window based on polycrystalline diamond, for improved brightness in microfocus X-ray tube was demonstrated. The polycrystalline diamond films which were deposited by hot filament chemical vapor deposition (HFCVD) approach revealed good intrinsic thermal properties. We developed a reliable method to fabricate and estimate the windows/target assembly, quantitatively analyzed the anode heat load of the X-ray source operating under the electron beam irradiation, and then tested in a microfocus X-ray tube. It's believed that the experiment and calculation results presented here can be generally applied for the design and the fabrication of a high brightness microfocus X-ray tube employing polycrystalline diamond windows.
\end{abstract}

KEYWORD: Microfocus X-ray source; Polycrystalline diamond film; Windows/target assembly; HFCVD; Anode heat load.

\section{INTRODUCTION}

Microfocus X-ray tube which generates X-ray with micrometer-sized focal spots have now been widely used (Heo S H, 2007, Jeong J.W, 2013). Since the spatial resolution of an X-ray image is mainly governed by the focal spot size of an X-ray source, a smaller focal spot is used readily for high resolution X-ray imaging (Jakubek J, 2006). For this purpose, transmission X-ray tubes are particularly well-suited (Yoshioka A, 2005). They are based on a windowtarget stack, ensuring the high geometric magnification of the sample. In the tube, a highly energetic focused electron beam impacts the target made of a high atomic number metal for producing bremsstrahlung. Besides the requirement of high x-ray transmission, the window needs to exhibit high mechanical strength and gas tightness for physical separation between the vacuum and the ambient air. The low atomic order number, high mechanical strength, and gas tight nature of thin beryllium foils allow the fabrication of beryllium x-ray transmission windows that satisfy the requirements, and make beryllium the most commonly used window material (Lynch D.R, 1996).

The source brightness is a key figure of merit for microfocus x-ray systems, which is proportional to the electron-beam power density at the anode (Yada K, 2009, Hemberg O, 2003). Therefore, application requirements impose increased electron-beam power density associated with punctual X-ray sources. As a consequence the rising of the electron-beam power density shed a new light on thermal issues occurring in the target/window system subjected to huge local heat densities. Typical electron-beam power densities can reach values as high as $10^{14} \mathrm{~W} / \mathrm{m}^{2}$, which almost entirely convert into heat in the target material (Cosslett V, 1952). In this case, beryllium exhibits limitations in dealing with such heat densities. On the contrary, diamond is a good candidate for such applications as it is composed only of carbon (low Z material with high X-ray transparency) and it has the highest known thermal conductivity of all bulk materials (up to $2000 \mathrm{~W} \cdot \mathrm{m}^{-1} \cdot \mathrm{K}^{-1}$ ) for single crystal diamond at room temperature (Delfaure C, 2015, Ying $X, 2000$ ). Diamond has Low thermal expansion resulting in low thermomechanical stress and no health risk. It has recently emerged as viable solution for transmission type X-ray generators with higher thermal performances. However, cost and size of natural diamond limited its practical applications. The recent developments in the field of low pressure vapor deposition technique for polycrystalline diamond films have made it as a practical useful material with reasonable cost in various applications (Reinhard D. K,2004). 
In this application, polycrystalline diamond films were fabricated by hot filament chemical vapor deposition method and then coated with the appropriate target material, which have been proved to be very useful and to improve the performance of the microfocus tube considerably. Here they have a triple function: they act as an X-ray-transparent vacuum window, a metal-coated target, and a heat sink for the heat generated by the electron beam. Moreover, we investigate the effect of several design parameters of a transmission-type diamond target on the Xray intensity and X-ray stability. From the analysis, we derive the optimum target thickness to achieve maximum X-ray intensity and present the maximum allowable electronbeam power loading for a stable operation of the X-ray target for incident electron energies at $100 \mathrm{keV}$.

\section{EXPERIMENT}

A HFCVD system was used to deposit optical quality diamond thin film. Polycrystalline diamond films were deposited by two steps (nucleation period and growth period) to keep high density of diamond nucleation and mufti-beam interference technology to monitor and accurately control the thickness of diamond thin film. This system has been depicted in detail previouslyx (Ali M, 2011). A gas mixture of $\mathrm{H}_{2}$ and $\mathrm{CH}_{3} \mathrm{COCH}_{3}$ was used as the precursor gases. The reactor pressure was varied from 0.5 Torr to 6 Torr. The temperature of the filaments was around $2100{ }^{\circ} \mathrm{C}$, as measured by an optical pyrometer through a quartz window. The deposition temperature was about $870{ }^{\circ} \mathrm{C}$, as measured by a thermocouple placed on the top surface of the tungsten disk, close to the Si wafer during the deposition. After stable and continuous deposition for more than $200 \mathrm{~h}$, diamond films with a thickness of $\sim 1.75 \mathrm{~mm}$ were obtained. The as-deposited diamond films were peeled off from substrate, then abraded by using commercial diamond grits and double-sided polished by fast rotating diamond grinding wheel. At last, the thickness of free-standing diamond films was 0.25 $\mathrm{mm}$ and surface roughness Ra was less than $10 \mathrm{~nm}$.

A solid-state bonding process can be used to attach windows with mounting flanges, and the bond must keep leak-tight and able to withstand a pressure differential of one atmosphere. The fabricated polycrystalline diamond films are mounted on the frame by vacuum brazing. Typically the window layer is covered with a few- $\mu \mathrm{m}$-thick layer of target material, e.g., tungsten in electrical contact with the frame and the protective ring in order to avoid charging of the dielectric diamond films. Tungsten anode targets were fabricated by magnetron sputtering method.

After the deposition and polish, the diamond films were characterized by X-ray diffraction (Rigaku Dmax X-ray diffractometer), scanning electron microscopy (Zeiss Supra40), studied using
Raman scattering excited by $632.8 \mathrm{~nm}$ laser light and UV Raman. NETZSCH Laser Flash Apparatus 447 was used to detect the thermal diffusivity of diamond films. A commercial helium leak tester (Pfeiffer HLT 570) is used to evaluate the helium leak rates of the fabricated diamond windows.

\section{RESULTS AND DISCUSSION}

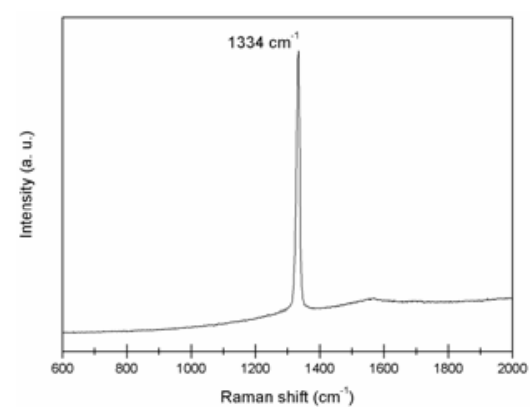

Figure 1. Raman spectra of the polycrystalline diamond films

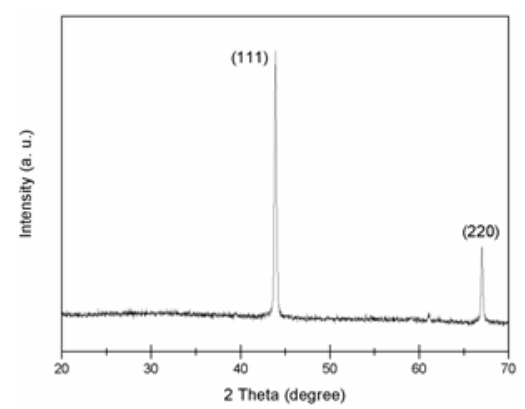

Figure 2. XRD pattern for the film

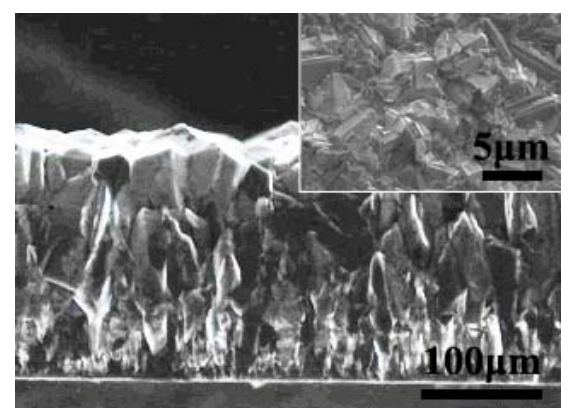

Figure 3. The cross-section and surface (inset) SEM images of the nanocrystalline diamond films

A Raman shift spectrum measured from one of the films deposited in this work is shown in Fig. 1. A sharp peak is observed at $1332 \mathrm{~cm}^{-1}$, indicating $\mathrm{sp}^{3}$ bond of diamond, which is well coincident with the position of Raman peak of nature diamond. Near $1500 \mathrm{~cm}^{-1}$, another much weak and broad Raman peak is attributed to a little amount of graphite and amorphous carbon deposited on the substrate with diamond. The coefficient of linear expansion of the monocrystal diamond having an ideal $\mathrm{sp}^{3}$ bonding frame structure as a principal component was 1.0 
$\mathrm{ppm} \cdot \mathrm{K}^{-1}$ at $20^{\circ} \mathrm{C}$, which is smaller than that of $\mathrm{sp}^{2}$ bonding frame structure $\left(3.1 \mathrm{ppm} \cdot \mathrm{K}^{-1}\right)$. Low thermal expansion of diamond with $\mathrm{sp}^{3}$ bonding frame structure resulting in low thermomechanical stress during a temperature rising.

As a XRD pattern of the as-obtained films, Fig. 2 can be calibrated to the diamond phase by contrast with the standard cards. The characteristic diamond peaks are evident in the pattern. Fig. 3 shows the SEM image of the specimen. It is observed that the specimen is polycrystalline diamond thin film. The sizes of the crystallites are around $5 \mu \mathrm{m}$.

The hermeticity level of the frame-installed window assemblies was tested with a He leak detector system. Helium leak rates below $2 \times 10^{-11} \mathrm{~Pa} \cdot \mathrm{m}^{3} \cdot \mathrm{s}^{-1}$ are considered sufficient to support the high quality vacuum throughout the life time of the detector. In addition, the helium leak rate is also evaluated after the diamond windows have been stressed with more than 100 pressure cycles of a differential pressure of one atmosphere in order to rule out micro crack formation during the cycle testing.

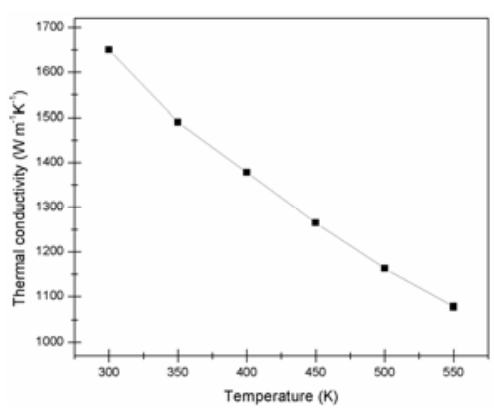

Figure 4. The through-thickness thermal conductivity of diamond films between $300 \mathrm{~K}$ and $550 \mathrm{~K}$

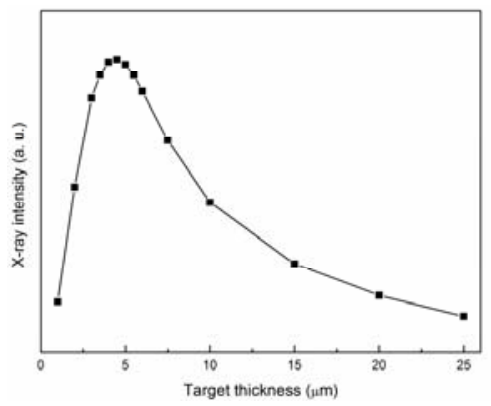

Figure 5. X-ray intensity as a function of thickness for W transmission-type targets with $100 \mathrm{keV}$ electron beam energy

The demand of diamond is putting forward to heat dispersion of target/window assembly. Fig. 4 shows the through-thickness thermal conductivity of diamond films from room temperature to $550 \mathrm{~K}$. It can be seen that the thermal conductivity $\kappa_{\perp}$ of diamond films reach up to about $1650 \mathrm{~W} \cdot \mathrm{m}^{-1} \cdot \mathrm{K}^{-1}$. It is well known that the main heat carrier in diamond is phonon, which would be scattered by phonon collision, defects and grain boundaries consequently re- ducing the mean free path of phonons. According to the previous report, the grain boundary scattering mechanisms are dominant only at low temperature (Klokov A, 2008, Verhoeven H, 1997), and weaken when temperature increases. So it is believed that grain boundary can be negligible when phonons propagate parallel to the horizontal axis of diamond film above $400 \mathrm{~K}$, and thus an isotropic thermal conductivity is used in the simulation model (Delfaure C, 2015).

In order to investigate the thermal stability of a transmission-type target, temperature profile inside the target/window assembly under the electron beam irradiation should be calculated. For the calculation of temperature profile, the optimum value of the target thickness deposited on the diamond window was firstly calculated using MCNP code (Ihsan A,2009); Fig. 5 shows a typical example for the X-ray intensity as a function of target thickness. In this particular example, the electron beam energy was $100 \mathrm{keV}$, and thus the optimum value of the target thickness at which X-ray intensity becomes maximum was about $5 \mu \mathrm{m}$ for $\mathrm{W}$ targets. In this work we have carried out transient thermal simulations using Ansys software, which is a powerful interactive environment for modeling and solving scientific and engineering problems based on partial differential equations. For the calculation simplification, it was assumed that an electron beam with a constant diameter of $5 \mu \mathrm{m}$ enters the centre of a circularly-shaped transmissiontype target with the diameter of $10 \mathrm{~mm}$, and the diamond thickness was $250 \mu \mathrm{m}$.
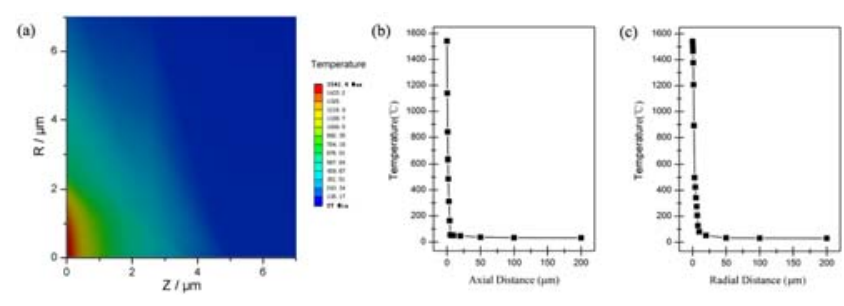

Figure 6. Temperature profiles for diamond transmission window for $100 \mathrm{keV}$ incident electron beam energy: (a) two dimensional axial-symmetric temperature distribution at target centre (b) axial and (c) radial temperature profiles for target/window assembly.

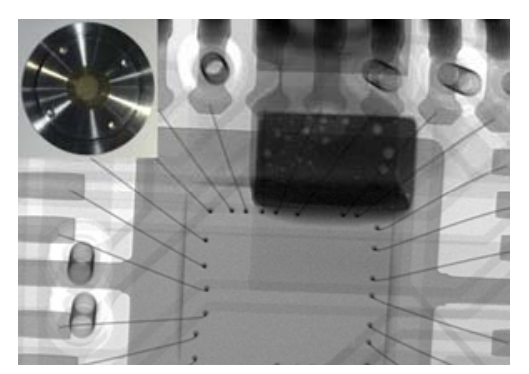

Figure 7. X-ray image of an IC board using polycrystalline diamond window. 
The simulations were carried out on a 2dimensional geometry having a cylindrical symmetry of the problem under steady state condition. The temperature of the circular peripheral region of the target was assumed to be maintained at room temperature throughout the simulation. Fig. 6(a) shows one example of the calculated results, which displays the two dimensional axial-symmetric temperature distributions of a $\mathrm{W}$ target. The incident electron beam current was $20 \mu \mathrm{A}$. The temperature was maximum to $1814.4 \mathrm{~K}$ on the surface of the target in the axial direction. The temperature decreased from the maximum value to about $350 \mathrm{~K}$ within $25 \mu \mathrm{m}$ in the radial direction and $20 \mu \mathrm{m}$ in the axial direction. This indicates that the temperature rapidly drops along both the axial and radial directions as shown in Fig. 6(b) and Fig. 6(c), respectively.

If we used the beryllium window in $200 \mu \mathrm{m}$ thick for calculation, and the other parameters were the same as described on diamond windows. The temperature was maximum on the surface of the target reached $2079 \mathrm{~K}$. From the perspective of thermal stability of X-ray tube, we calculated the maximum allowable electron beam current that does not melt the target/window assembly. Through repeatedly calculation on the temperature profile by changing the beam current impinging on the target at fixed beam energy, we finally find a certain beam current $(50 \mu \mathrm{A} @ 100 \mathrm{kV})$ at which the maximum temperature of target/window assembly reached the melting point of a target material (W: $3695 \mathrm{~K}$ ).

Imaging, is a good test of the system stability and performance since any change of the source during the exposure will degrade the image quality. Figure 7 depicts two typical images captured using the diamond window assembly for the x-ray generation. The contrast and resolution is good and shows that the polycrystalline diamond window is very suitable for imaging of transmission microfocus $\mathrm{x}$-ray source.

\section{CONCLUSION}

In this work, the suitability of polycrystalline diamond film as a window material for $\mathrm{x}$-ray transmission windows has been demonstrated by equipping a vacuum encapsulated microfocus X-ray tube. Polycrystalline diamond films have been grown using a gas mixture of hydrocarbon and $\mathrm{H}_{2}$ by HFCVD method. The fabricated diamond windows were characterized in detail, and revealed good strength and gas tightness. Calculations and simulations on the thermal performance under electron beam impacts the targets were made. The simulations indicated that diamond window/target assembly has high tolerance against high electron beam power. These properties combined with the demonstrated uniformity, repeatability and high X-ray transparency make the polycrystalline diamond based X-ray transmission windows very attractive and a reliable choice to replace traditional beryllium based X-ray windows.

\section{ACKNOWLEDGMENT:}

This work was sponsored by the National Natural Science Foundation of China (NSFC) (No. 51301155). The authors deeply appreciated their financial supports.

\section{REFERENCES}

Ali M., Ürgen M., Surface morphology, growth rate and quality of gas pressures. Appl. Surf. Sci., 2011, 257(20):84208426

Cosslett V., Nixon W., X-ray shadow microscopy, Nature 170 (1952) 436-438.

Delfaure C., Mazellie J. P., Tranchant N., Bergonzo P., Ponard P., Saada S., Nanofocus diamond X-ray windows: Thermal modeling of nano-sized heat source systems, Diam. Rela. Mater. 59 (2015) 104-115.

Hemberg O, Otendal M, Hertz H M. Liquid-metal-jet anode electron-impact X-ray source. Appl. Phys. Lett., 2003, 83(7):1483-1485.

Heo S H, Ihsan A, Cho S O, Transmission-type microfocus Xray tube using carbon nanotube field emitters. Appl. Phys. Lett., 2007, 90(18):183109-183112.

Ihsan A, Heo S H, Cho S O. A microfocus X-ray tube based on a microstructured X-ray target. Nucl. Instru. Meth. Phys. Res. B, 2009, 267(21):3566-3573.

Jeong J.W., Kang J.T., Choi S., et al. A digital miniature X-ray tube with a high-density triode carbon nanotube field emitter. Appl. Phys. Lett., 2013, 102(2): 023504.

Jakubek J, Holy T, Jakubek M, et al. Experimental system for high resolution X-ray transmission radiography $(\mathrm{J})$. Nucl. Instru. Meth. Phys. Res. A, 2006, 563(1): 278-281.

Klokov A., Aminev D., Sharkov A., Ral'chenko V., Galkina T., Thermal parameters of layers and interfaces in siliconon-diamond structures, Phys. Solid State 2008, 50 (12):2263 -2269 .

Lynch D.R., Berman L., Montanez P., Pjerov S., Stefan P., Woodle M., Beryllium windows for synchrotron light sources, SPIE, 1996, 2855:119

Reinhard D. K., Grotjohn T. A., Becker M., Yaran M. K., Schuelke T., Asmussen J., Fabrication and properties of ultranano, nano, and microcrystalline diamond membranes and sheets, J. Vac. Sci. Technol. B, 2004, 22(6):2811-2814

Verhoeven H., Boettger E., Flöter A., Rei H., Zachai R., Influence of the microstructure on the thermal properties of thin polycrystalline diamond films, Appl. Phys. Lett., 1997, 71(10):1329-1332.

Yoshioka A, Yamaguchi Y, Tamura K, et al. Monte-Carlo simulation of spatial distribution of X-rays in multi-film targets. II: Spatial distribution of continuous x-rays and temperature elevation in W/Al film targets. Surf. Inter. Anal., 2005, 37(3):356-361

Yada K. Recent trends of projection X-ray microscopy in Japan, Spectrochimica Acta Part B, 2009, 64(8):729-735.

Ying X., Xu X., CVD diamond thin film for IR optics and Xray optics, Thin Solid Films, 2000, 368(1):297-299. 